\title{
Intelligent Identification Technology of Attributes of Users' Transformers Based on Gray Correlation Analysis
}

\author{
Yan Liu, ${ }^{1 *} \mathrm{Hu}$ Yue, ${ }^{2}$ Yang Feng, ${ }^{3}$ \\ Hongying Miao, ${ }^{4}$ Sida Zhen, ${ }^{1}$ and Chih-Cheng Chen ${ }^{5,6^{* *}}$ \\ ${ }^{1}$ Electric Power Research Institute, State Grid Jibei Electric Power Co., Ltd., Beijing 100045, China \\ ${ }^{2}$ Department of Marketing, State Grid Jibei Electric Power Co., Ltd., Beijing 100032, China \\ ${ }^{3}$ Qinhuangdao Power Supply Company, State Grid Jibei Electric Power Co., Ltd., \\ Qinhuangdao, Hebei 066000, China \\ ${ }^{4}$ Chengde Power Supply Company, State Grid Jibei Electric Power Co., Ltd., Chengde, Hebei 067000, China \\ ${ }^{5}$ Department of Aeronautical Engineering, Chaoyang University of Technology, Taichung 413310, Taiwan \\ ${ }^{6}$ School of Information Engineering, Jimei University, Xiamen, Fujian 361021, China
}

(Received September 6, 2020; accepted March 1, 2021)

Keywords: attributes of users' transformer identification, gray correlation analysis, intelligent identification, correlation

Power grid construction and power measurement automation systems are gaining popularity and becoming ever more commonplace in developing countries. However, adoption rates are affected by inaccurate intelligent identification systems that control remote meter reading and line loss management. Local electricity distribution networks also have inconsistent user cable wiring, different geographical topologies, and issues with cable crosstalk, which lead to inaccurate readings of users' data coming from the intelligent identification system, and sometimes even a failure to read electricity meters. Taking into consideration the voltage required for an intelligent identification system, we propose a new transformer for an intelligent identification system. Our new transformer improves the gray correlation analysis of watt-hour meters, which overcomes the shortcomings of existing identification methods by evaluating line voltages between unidentified and identified watt-hour meters. Experimental results show that our transformer with this method can accurately measure users' electricity meter readings and perform line loss management.

\section{Introduction}

The continual development and improvement of power grids has greatly improved the distribution network and infrastructure of power grids in developing countries. However, the traditional power management methods have become increasingly incompatible and inefficient with the advent of newer connected technologies. To resolve this deficiency, there is a strong focus on the precise management of transformers that assist in electric meter readings and line loss management. In a world of accurate line management, precise user electric data can resolve many power management deficiencies and improve logistics. The improvement of such readings

\footnotetext{
*Corresponding author: e-mail: vipliuyan1989@126.com

*** Corresponding author: e-mail: ccc@gm.cyut.edu.tw

https://doi.org/10.18494/SAM.2021.3159
} 
can also significantly improve the logistics of assets deployed and managed and the reliability of the power distribution network. Failed electric meter readings are mainly a result of differing correlation information records, incorrect online updates, incomplete statistical information, and other reasons. They also affect dispute resolution processes as a result of different measurements from affected parties, which leads to adverse relations between users and power companies. Therefore, it is important to ensure that the transformers of smart meter monitoring systems are accurate. Existing electric meter measurement is dependent on field testing by power supply employees, in which workers manually check that the transmission path is as it should be and that lines are associated with the correct corresponding transformer. This current model involves a large number of field personnel and is unsuitable for detecting underground cable malfunctions. Since the detection of underground cable malfunctions is crucial for power management, we proposed a transformer region monitoring system that implements current pulses to detect line loss, similar to systems stated in the literature. ${ }^{(1-3)}$

However, high voltages, crosstalk, and the mutual grounding of cables result in large signal interference. ${ }^{(4)}$ To solve the potential security issues resulting from the operation of a pulse current method, we utilize a back-propagation (BP) neural network to match household variables from users' data, ${ }^{(5)}$ which can supplement data attached to the carrier signal. The signal quality also indicates the region of the transformer. In addition, large infrastructure differences in routing branches and network topologies in different areas, as well as long transmission distances, make it difficult to accurately identify user signals.

Ye et al. proposed intelligent identification technology of household transformers focusing on the attributes of users' transformers and data clock correlation. ${ }^{(6)}$ They used data mining to categorize the acquisition equipment required in each transformer area. Then, they classified regional membership in the event of an unrecognized acquisition. However, this implementation process is extremely complex and difficult to implement. Duan et al. put forward the idea of evaluating survey data for a smart watt-hour meter that is connected to gray relational data to recognize the attributes of users' transformers and the phase of the platform area. ${ }^{(7)}$ Because the weight of each variable is fixed in the correlation calculation and also subjective, the reliability of the results is low in a complex environment. Yu et al. proposed the first-order differential attributes of users' transformers of the line loss rate as an index to diagnose abnormalities in users' transformer attributes, ${ }^{(8)}$ and abnormalities were captured under the constraint of the proximity relationship of the common change space.

We have taken the useful features from the above paradigms and implemented them in our system. Through extensive testing and application in a sample area, our system can efficiently distinguish household variables and provide an effective reference for on-site verification of households. Additionally, the collection and copying of this power data can be combined with other technologies such as instantaneous freezing systems. Ping et al. proposed a method of checking the low-voltage topological relationship of the distribution platform area. ${ }^{(9)}$ In order to solve the problem of low accuracy of the attributes of users' transformer identification, State Grid Corporation of China launched intelligent users' transformer identification based on the meter high-speed power line communication (HPLC) technology in 2018. In this technology, the concentrator broadcasts the identification characteristic signal on the low-voltage line in the 
distribution station area. The user's transformer receives the characteristic signal, then records the characteristic code of the station area, and finally sends the transformer file number and the station area characteristic code to the concentrator. This paper provides a new technical solution for realizing the quick, accurate, and complete discrimination of files in the platform area, without too many omissions and false positives, reducing the need for manual labor and improving the timeliness of the identification and checking of the platform area, which has important engineering value. Using the narrowband Internet of Things (IoT) and the emerging machine learning (ML) support vector machine algorithm, a novel and intelligent way of identifying the attributes of users' transformers is proposed. First, it is used to upload the voltage data volume to the host computer with the help of the narrowband IoT (NB-IoT) communication paradigm. Through equation computation and data analysis, it was found that the voltage data of the power collection equipment in the same area has a spatiotemporal correlation. Given this situation, the space correlation and voltage-time correlation are used as feature factors, and a support vector machine category solution is used for judging the attribute relationship of the smart device to be identified. In addition, the identification paradigm can provide users with accurate attributes of their transformers, finally realizing efficient management of the attributes of users' transformers.

On the basis of our results, ${ }^{(7)}$ an improved gray correlation analysis technology for recognizing users' transformer attributes in intelligent platform areas is proposed. We introduce the concept of entropy into this method to compute the correlation, thus eliminating some subjective estimation. The correlation of the converged line voltage can not only be used to recognize the transformer region but also escape the negative impact of low-grade carrier signal communication. In addition, the implementation process is extremely simple. The precision of identification in sophisticated environments provides a technical improvement for effective management. A modified gray correlation analysis method is proposed for area recognition. The main highlights are as follows. The voltage eigenvalues for the intelligent attributes of users' transformers are used to perform a gray correlation evaluation with the goal of household identification of users' transformer areas, which will overcome the problems of user wiring modes, the topology structure, and communication crosstalk. The core idea of this method is to establish the relationship between cross-area or unidentified electricity meters and identify electricity meters in terms of line voltage eigenvalues.

The rest of this paper is organized as follows. The correlation of smart meter data is first given in Sect. 2. Section 3 explains the gray correlation algorithm analysis in detail, which contains traditional gray correlation analysis and an improved gray relational analysis algorithm. In Sect. 4, smart identification of the attributes of users' transformers is described, then results and discussion are given in Sect. 5. Section 6 concludes this paper.

\section{Intelligent Identification Design of Attributes of Users' Transformers}

At present, the attributes of users' transformer identification technology include two categories: traditional manual transformer area identification and special transformer area identification instruments. More specifically, manual transformer area identification mainly 
depends on personnel visiting the site to check the transformer area attributes, which is inefficient and time-consuming. In recent years, the development and use of special user transformer area identification equipment have greatly improved the identification speed. The existing users' transformer identification technology mainly adopts a carrier communication method and current pulse paradigms. However, misidentification often occurs with the special users' transformer identification instrument based on the carrier communication paradigm because the carrier signal cannot be completely isolated by the transformer in practical applications. The current pulse paradigm is a promising solution for allowing the users' transformer identification device to identify the attribute by sending out a high-frequency pulse signal.

The household users' transformer recognition device includes a change table relationship recognition device and a household table relationship recognition device. The device can be used frequently without affecting the user's normal electricity consumption as shown in Fig. 1. At the same time, it does not need to check each meter box and can identify all electrical and physical connections at one time, but changes the table correspondence relationship, identifies all the electric meters installed in the station area, and correctly distinguishes the household table affiliation. The hardware of the system includes a meter and a transmitter, which can be used in two scenarios. (1) Variable table relationship recognition: When the device performs variable table relationship recognition, the meter finder is connected to two areas, e.g., areas $\mathrm{A}$ and $\mathrm{B}$, at the platform concentration. By power line carrier communication, the algorithm process of meter seeking is implemented to automatically collect all the meter numbers in the desk area, identify the phase line where the meter is located, and display the meter number and phase on the display. (2) Identification of attributes of users' transformer relationship: When the device is used for identification of the household meter relationship, the transmitter with the carrier module is placed in the user's home, and the room number of the user is continuously transmitted. The meter receives the room number at the outlet of the user's electricity meter. If the room number is received, the membership relationship of the household meter can be determined and displayed on a human-machine interface.

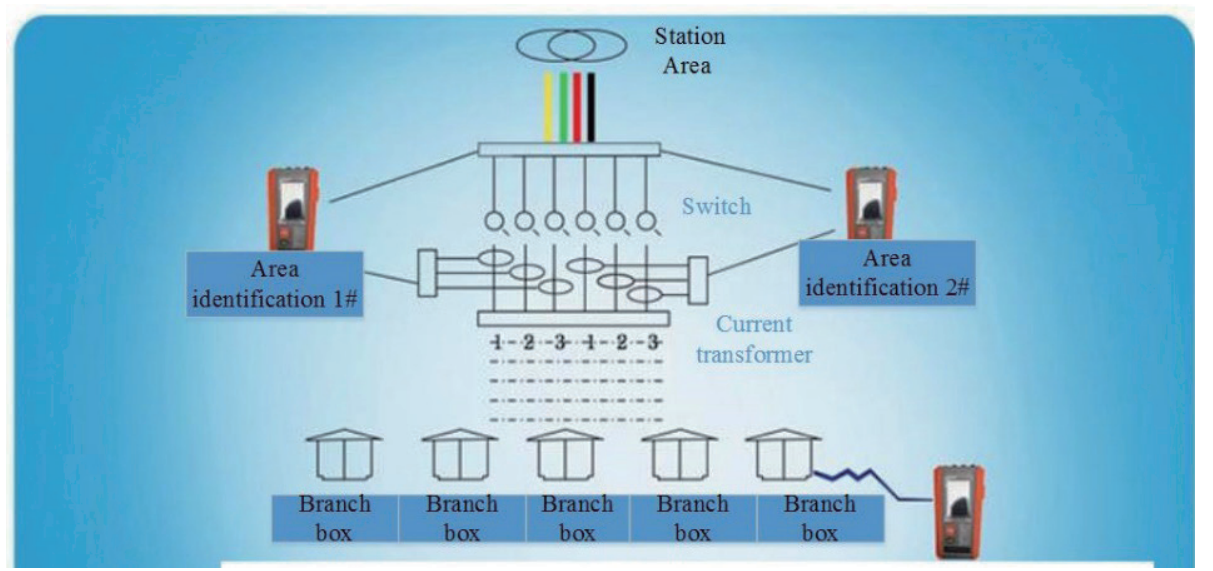

Fig. 1. (Color online) Household identification design of intelligent attributes of users' transformers. 


\section{Correlation of Smart Meter Data}

It is well known that the network topology of terminal devices in the current transformer region is anthropomorphic, which means that the working capacity communicated to the current network paradigm changes over time. As a result, the line voltage often fluctuates. If and only if the meter remains in a uniform transformer area, the line voltage fluctuation direction converged by the meter will remain similar because the continuous output of the transformer terminal is the same. In addition, the electrical transmission distance is comparatively short, as shown in Fig. 2. Readers interested in smart meter data correlation can refer to Refs. 10 and 11.

When the meter is located in different transformer areas, the distance is relatively long because the terminal output of the transformer is different, and the line voltage fluctuation trend collected by the meter is also different, as depicted in Fig. 3.

\section{Gray Correlation Algorithm Analysis}

The paradigm of introducing gray correlation analysis into pattern recognition has been recently receiving significant attention because its computational complexity is lower than that of most existing statistical paradigms. The recognition result can generally accomplish the corresponding prototype of the proposed promising algorithms, i.e., neural networks, and facilitate vector machines. This paradigm can be used for the identification of smart watt-hour meters. From the viewpoint of the system, known sampling information is used to study the relationship between the information and reveal unknown correlation information. The existing transformer district should be assumed to be a sophisticated gray architecture. However, some existing power meters (known as accepted electric energy meters) as well as their accepted measurements are given information. In addition, neither the unrecognized area nor cross-zone meters should guarantee information sets. At the same time, from the perspective of sampling information and system architecture, the "whitening paradigm" transformation of the gray

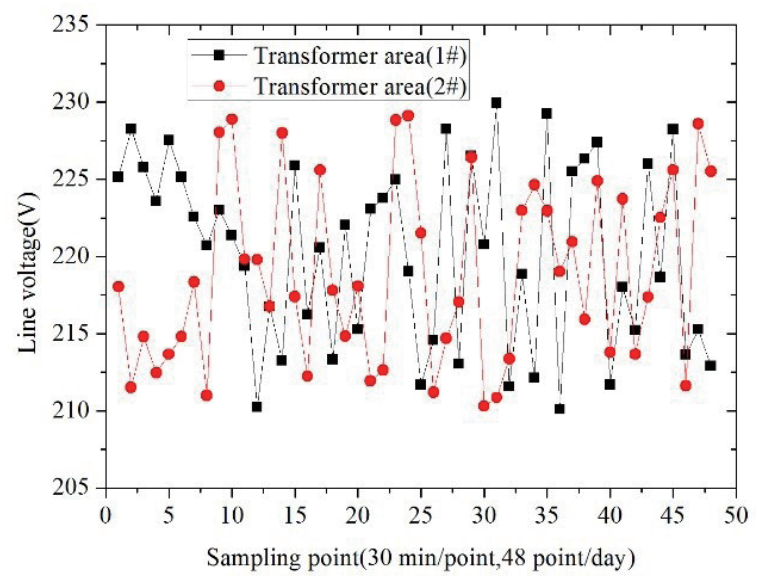

Fig. 2. (Color online) Voltage meter diagram of attributes of users' transformers for area A.

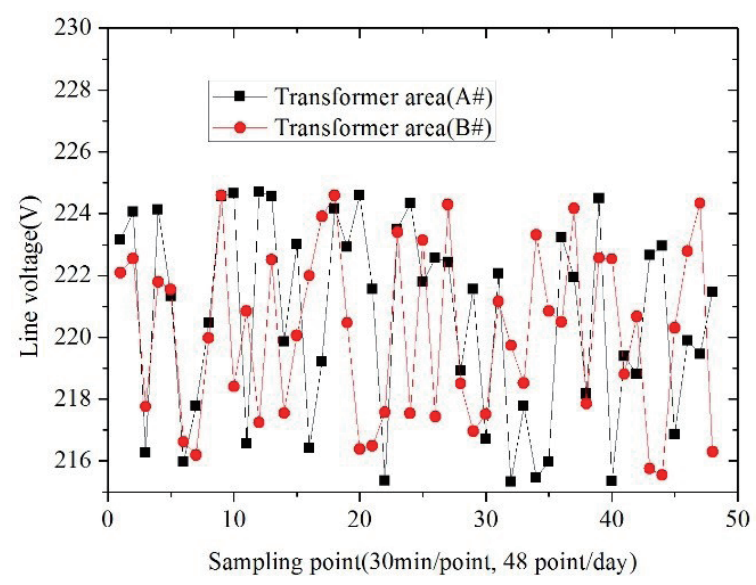

Fig. 3. (Color online) Voltage meter diagram of attributes of users' transformers for areas A and B. 
system represents the identification process of the transformer region, and the correlation degree is used to represent the correlation degree of the analyzed object. The gray relational dissolution is generally used to express the grade of correlation of the described objects provided by the degree of correlation. The target of this paradigm is used to obtain the key relationship between the identified power transformer watt-hour meter and the unidentified power transformer watthour meter. Then, the actual area of the household transformer can be found. The following analysis illustrates the application of gray correlation analysis in area recognition.

\subsection{Gray correlation analysis}

The line voltage relationship and correlation grade between unrecognized and recognized watt-hour meters are determined by using a gray correlation analysis algorithm. The greater the correlation, the closer the distance between the two meters, and vice versa. References 12 and 13 show the correlation analysis process. The reference sequence $N_{0}$ and comparison sequence $M$ are used to represent the line voltage characteristics of the unrecognized and recognized watt-hour meters at different times, respectively.

$$
\left\{\begin{array}{c}
N_{0}=\left[N_{0}(1), N_{0}(2), \ldots, N_{0}(n)\right] \\
M_{j}=\left[M_{j}(1), M_{j}(2), \ldots, M_{j}(n)\right]
\end{array}\right.
$$

Here, $n$ is the total number of the voltage characteristic values and is known as the characteristic amount in the defined sequence model. Here, if $n$ is equal to $k$, then parameter $k$ defines the $k$ th eigenvalue of this defined sequence model. Parameter $J$ represents the $j$ th approved meter. The correlation coefficient calculation between the recognized watt-hour meter and the unidentified meter factor in terms of the voltage characteristic value is as follows:

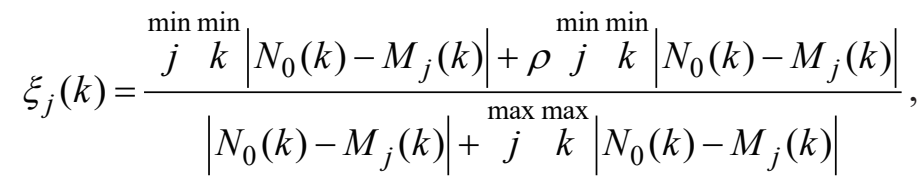

where the resolution coefficient $\rho$ varies from 0 to 1 and is usually set as 0.5 . $\xi_{j}(k)$ is the correlation parameter coupling factor between $N_{0}$ and $M_{j}$, and the constraint $1 \leq k \leq n$ exists. Because of the enormous number of correlation parameters, the attributes of users' transformer identification are not easy to distinguish, and the gray correlation grade is often used to represent the correlation between the defined sequence $M_{j}$ and the reference sequence $N_{0}$, which can be expressed by $\lambda_{j}$.

$$
\lambda_{j}=\frac{1}{n} \sum_{k=1}^{n} \xi_{j}(k)
$$

Since the weight parameter $1 / n$ in Eq. (3) is defined and individual, the effect of individual sampling points on the determination of witness marks should be given priority. The basic 
method does not meet the specific requirements for the identification of users' transformer attributes in the basic users' transformers area, because the characteristic value of the voltage (overload, load, etc.) changes with time in the calculation of the gray relational degree. Therefore, the concept of entropy is introduced to improve the gray relational analysis, and the algorithm used is described in detail in Sect. 4.2.

\subsection{Improved gray relational analysis algorithm}

The uncertainty of the system in the energy region is described by information entropy, where larger numbers indicate more indetermination in the proposed network architecture. Here, we introduce an entropy function that is conducive to gray relational analysis and effectively reduces the individual judgment from formation and analysis. ${ }^{(14,15)}$ Although several gray relational degree calculation methods have been proposed to determine the degree of correlation between gray system factors, none of them are perfect for the following two reasons. First, the purpose of dimensionless processing of the original sequence of numbers is to facilitate a comparison between different dimensions and orders of magnitude, and it should not distort the internal relations between factors or conceal the true nature of things. Second, normalization is one of the four axioms of gray correlation (also known as the four principles or the four characteristics). It first stipulates that the interval of the gray correlation is $(0,1) .{ }^{(16)}$ Considering that things are always the same in relation to themselves, the correlation is less than or equal to 1 . The detailed steps are as follows:

(1) The setting $\Delta_{j k}=\left\{N_{0}(k)-M_{j}(k)\right\}$ confirming the initial matrix $\Delta$ is

$$
\Delta=\left(\Delta_{j k}\right)_{m \times n}=\left[\Delta_{j 1}, \Delta_{j 2}, \Delta_{j 3}, \ldots, \Delta_{j n}\right]
$$

where parameter $n$ represents the sampling number of the voltage eigenvalue, $m$ is the number of unapproved meters, both $j=1,2, \ldots, n$ and $k=1,2, \ldots, n$ exist, and $\Delta_{j k}$ defines the absolute correction value of the $k$ th relevant eigenvalue between the $j$ th unidentifiable meter attribute and the identifying voltmeter.

(2) The proportional probability value of the recent occurrence of the $k$ th voltage eigenvalue presented for the $j$ th unidentified meter is $p_{j k}$.

$$
p_{j k}=\Delta_{j k} / \sum_{k=1}^{m} \Delta_{j k}
$$

(3) Extensiveness of the voltage eigenvalue $k$

From a statistical viewpoint, the differences between meters can be reflected by large deviation characteristics. Thus, the more diverse the eigenvalues are, the more crucial the meter is. Owing to the deep understanding of the redundancy concept in information theory, it is easy to define the redundancy of the $k$ th voltage characteristic value, which is established as follows:

$$
D_{k}=1-e_{k}
$$




$$
\begin{gathered}
e_{k}=E_{k} / E_{\max }, \\
E_{k}=-\sum_{j=1}^{n} p_{j k} \ln p_{j k}, \\
E_{\max }=\ln M,
\end{gathered}
$$

where $e_{k}$ represents the relative entropy value of the $k$ th voltage eigenvalue, $E_{k}$ indicates the entropy function, and $E_{\max }$ refers to the maximum entropy. Equation (1) is the relative entropy subtracted from one, which is the difference between the optimal entropy term and the $k$ thorder entropy term. The greater the weight $D_{k}$, the greater the significance of the representative entropy, which should be given a larger weight value.

(4) Weight value $a_{j}(k)$ of the characteristic value of the voltage $k$

$$
a \square k=D \quad \sum_{k=1}^{m} D
$$

This clearly leads to $\sum_{k=1}^{n} a_{j}(j)=1, a_{j}(j) \geq 0$.

(5) Correlation grade of enhanced gray correlation presentation $\gamma_{j}$

By replacing the fixed weight value $1 / n$ in Eq. (3) with the variable weight $a_{j}(k)$, we obtain

$$
\gamma_{j}=\sum_{k=1}^{n} \xi_{j}(k) a_{j}(k)
$$

where the closer $\gamma_{j}$ in Eq. (8) is to 1, the higher the correlation value between the identified meter and the unidentified meter. By comparing the correlation between the identified meter and the unidentified meter, the region in which the unrecognized meter belongs can be obtained.

\section{Results and Discussion}

In this part, we will use the improved gray correlation analysis in a real intelligent identification example. The steps are as follows: (1) store and collect the line voltage curve parameters of the currently identified and unidentified electricity meters, (2) classify the correlation between identified and unidentified electricity meters to improve the gray correlation decomposition paradigm, and (3) determine the correlation grade of the enhanced gray correlation presentation and break down the association relationship to determine the region of domestic transformers.

\subsection{Results}

A total of 15 electricity meters were applied for approval in the following two attributes of users' transformers, as shown in Fig. 4. However, both $1 \#$ and $4 \#$ in zone A, as well as both 


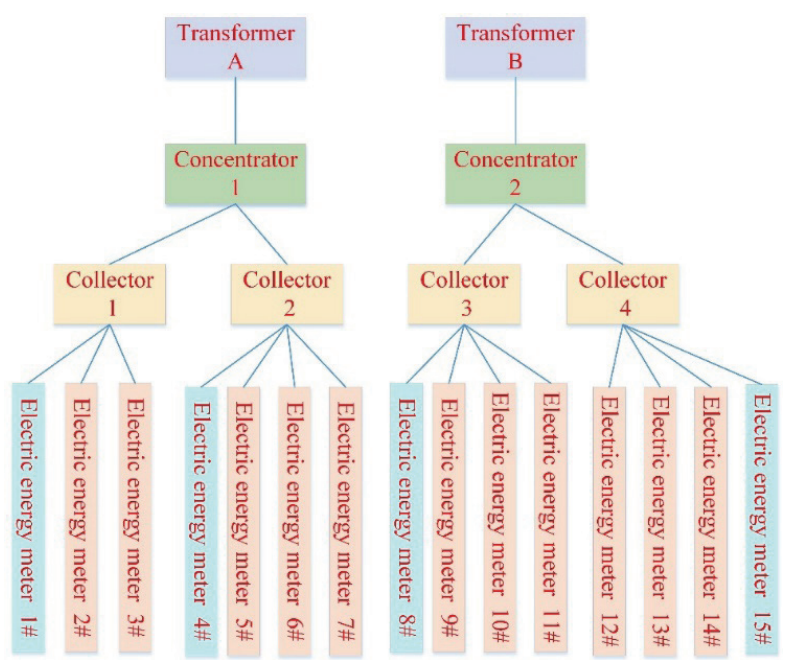

Fig. 4. (Color online) Architecture of attributes of users' transformers.

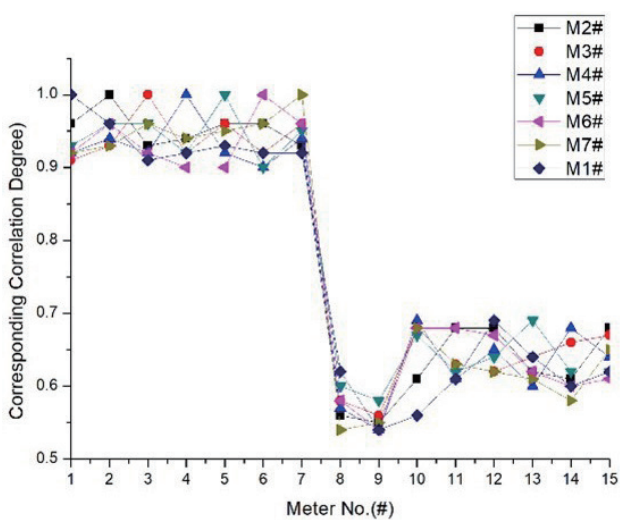

(a)

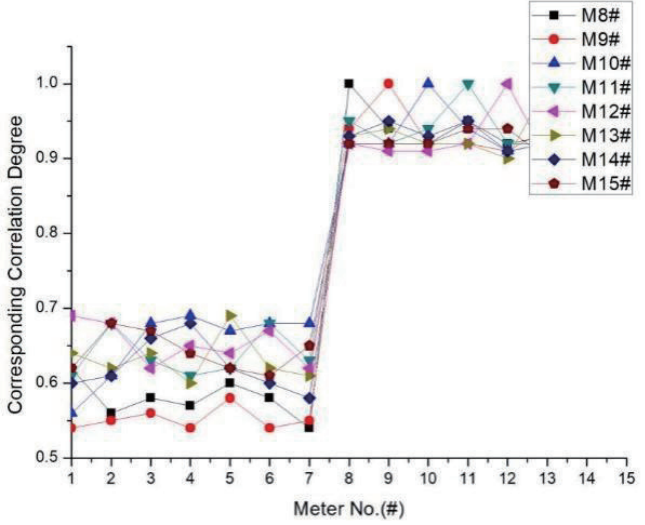

(b)

Fig. 5. (Color online) Related results of attributes of users' transformers A (a) and B (b) watt-hour meters.

$8 \#$ and $15 \#$ in zone B, are identified meters. The residual meters are those that need to be identified. The modified gray correlation presentation is made on $15 \mathrm{~m}$ of line voltage data. The corresponding correlation data are listed in Fig. 5.

As can be clearly seen from Fig. 5, the correlation grade of electricity meters deployed in the same region is very high (in 15 cases of meter application approval in two attributes of users' transformers, the correlation grade is greater than 0.90). However, the correlation value between electricity meters deployed in individual regions is considerably smaller (less than 0.70 ). This is because different electrical distances and load distributions lead to different degrees of correlation in different areas. The electrical distances between the two identification areas presented in this paradigm are insufficient, whereas the user selection types are very similar. In addition, the two areas have similar electricity consumption behavior according to an advanced 
correlation analysis. However, there is sufficient data in this envisioned paradigm to identify the region of the transformer. In addition, the correlation between the watt-hour meters in different transformer areas is low in other cases. Based on the previous discussion, \#1 and \#4 in users' transformer area A and $8 \#$ and $15 \#$ in area B are the accepted meters. The transformer area of the unidentified meter can be easily determined by using the correlation. According to the results in Fig. 4, voltmeters $1 \#-7 \#$ and voltmeters $8 \#-15 \#$ belong to two different intelligent platform regions, and the accuracy rate of the identification technology is close to $100 \%$. The results show that the improved gray correlation analysis proposed in this work can be performed exactly and in the transformer area.

\subsection{Discussion}

In general, errors in the customer relationship in the attributes of users' transformers are caused by the line reconstruction of voltage eigenvalues, and the account data are not updated in time. Errors in the customer relationship generally occur in the adjacent area of users' transformers. In order to avoid adjusting the overall household identification in the variable relationship, it is necessary to adjust the household identification in the variable relationship under the constraint of the spatial proximity relationship. For this purpose, spatial proximity relationships need to be maintained for each area of abnormal users' transformers. The adjacent table areas of each attribute of users' transformers are calculated using the spatial geographic coordinates of the table area, and the information of these table areas is saved. In the actual test data report (TDR), ${ }^{(17)}$ testing in only these 15 users' transformer areas can reduce the amount of calculation in the algorithm and avoid the occurrence of abnormal results. When testing the proposed platform, an abnormal user should be adjusted to the relationship, and the influence of the abnormal user on the line loss of the adjacent platform should also be calculated using Eqs. (1) and (2). We add the abnormal user's electric quantity data to the new attributes of users' transformers to calculate the new line loss fluctuation. If the fluctuation decreases, the user belongs to the new users' transformers area. More specially, we define some priorities. Priority 3: The average line loss rate is less than 0.04 , or negative; Priority 2: Line loss ratio is greater than 0.06; Priority 1: Line loss fluctuation is greater than 0.2; Priority 0: line loss fluctuation and line loss rate are in the normal range. The identification technology of intelligent attributes of users' transformers based on improved gray correlation analysis is significantly better than previous methods in terms of the line loss rate for the same priority.

\section{Conclusion}

A new method that is based on the analysis of conventional and existing users' transformer attribute identification technology is proposed to improve the gray correlation analysis. By considering the gray relational degree, the introduction of the gray relational degree entropy can be used to calculate the relational grade. Through detailed analysis of the strong line-voltage correlation of the watt-hour meters in the transformer region, the watt-hour meters deployed in the transformer region can be identified. Numerical examples show that the analysis results are accurate and have high practical value under complex environmental conditions. 


\section{Acknowledgments}

This work was supported by National Natural Science Foundation of China (Grant 51577028).

\section{References}

1 C. Y. Zhi, S. Yu, and Y. Fan: 2019 IEEE Sustainable Power and Energy Conf. (iSPEC, 2019) 629-633.

2 Z. Liu, Z. Dai, and P. Yu: 2019 IEEE 28th Int. Symp. Industrial Electronics (ISIE, 2019) 1827-1832.

3 C. Liu, J. Wang, and M. Wu: 2019 IEEE Innovative Smart Grid Technologies-Asia (ISGT Asia, 2019) 41374141.

4 J. Xue and T. T. Wang: Com. Eng. Appl. 22 (2017) 344. https://doi.org/10.1016/j.ijepes.2014.06.025

5 W. U. Shuang, H. U. Wei, and D. Yu: 2019 IEEE Innovative Smart Grid Technologies-Asia (ISGT Asia, 2019) 3624-3628.

6 A. Ye, J. Gu, and X. Zhang: Modern. Elect. Tech. 4 (2018) 30. https://doi.org/10.16652/j.issn.1004373x.2018.04.008

7 J. Duan, Y. He, and B. Du: IEEE Access 7 (2019) 62705. https://doi.org/10.1109/ACCESS.2019.2916461

8 H. Yu, S. B. Xia, and W. Wei: Elect. Measure. Tech. 42 (2019) 32. https://doi.org/10.19651/j.cnki.emt.1802250

9 L. Ping, H. Y. Yong, and Z. X. Ming: 2019 IEEE Innovative Smart Grid Technologies-Asia (ISGT Asia, 2019) $2550-2554$.

10 J. O. Sinayobye, S. K. Kyanda, and N. F. Kiwanuka: 2019 IEEE/ACM Symp. Software Engineering in Africa (SEiA, 2019) 1-10.

11 W. Jiang, J. Chen, and H. Tang: IEEE Trans. Smart. Grid. 10 (2019) 6965. https://doi.org/10.1109/ TSG.2019.2936148

12 Q. Z. Wang, J. K. Liu, and X. X. Zhu: Cold. Reg. Sci. Tech. 126 (2016) 44. https://doi.org/10.1016/ j.coldregions.2016.03.003

13 W. Q. Xiong, L. Liu, and M. Xiong: Clean. Tech. Env. Policy. 12 (2016) 401. https://doi.org/10.1007/s10098009-0214-7

14 Y. Qi, R. Huang, and H. Wu: 2019 IEEE Innovative Smart Grid Technologies-Asia (ISGT Asia, 2019) 14911496.

15 L. Zhou, Y. Wang, and F. Wang: 2017 IEEE Electrical Insulation Conf. (EIC, 2017) 491-494.

16 G. Dong, W. Wei, X. Xia, M. Woźniak, and R. Damaševičius: Electronics 9 (2020) 130. https://doi.org/10.3390/ electronics 9010130

17 Z. Zhang and B. W. Schafer: CFSRC Reports (2020). http://jhir.library.jhu.edu/handle/1774.2/62850

\section{About the Authors}

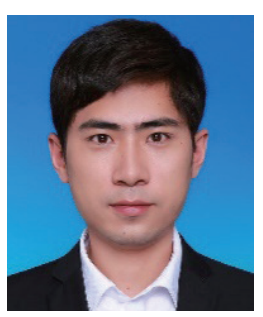

Yan Liu received his M.S. degree from Harbin Institute of Technology, China, in 2014. He then joined Electric Power Research Institute, State Grid Jibei Electric Power Co., Ltd., Beijing, China, where he is currently an engineer. His research interests include electricity information collection and electricity metering. (vipliuyan1989@126.com)

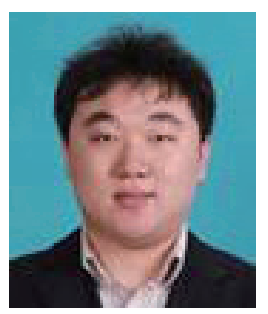

Hu Yue received his B.S. degree from Hebei University of Technology, China, and his M.S. degree from Yanshan University, China, in 2009 and 2015, respectively. He is an engineer at the Department of Marketing, State Grid Jibei Electric Power Co., Ltd., Beijing, China. His research interests are marketing project management, electricity information acquisition, power metering, line loss management, and connecting sales and distribution data. (Yuehu4045116@163.com) 


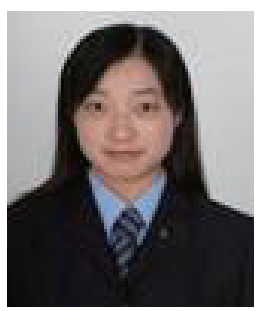

Yang Feng received her B.S. degree from Southwest University of Science and Technology (SWUST), China, in 2006. She is an engineer at Qinhuangdao Power Supply Company, State Grid Jibei Electric Power Co., Ltd., Qinhuangdao, Hebei, China. Her research interests are electricity information collection and electricity metering. (fengyangworkyxb@163.com)

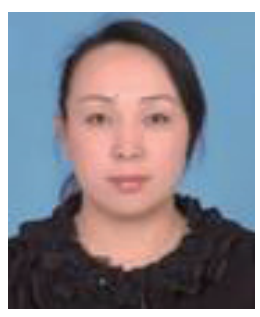

Hongying Miao received her B.S. degree from Hebei University of Technology, China, in 2010. She is a senior engineer at Chengde Power Supply Company, State Grid Jibei Electric Power Co., Ltd., Chengde, Hebei, China. Her research interests are electricity information collection and electricity metering. (miaohongying1977@126.com)

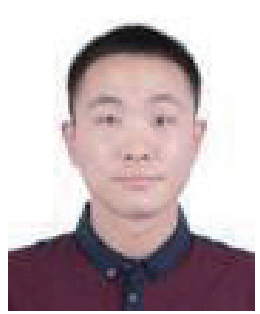

Sida Zhen received his M.S. degree from Shanghai Jiaotong University, China, in 2016. He is an engineer at Electric Power Science Institute, State Grid Jibei Electric Power Co., Ltd., Beijing, China. His research interests are electricity information collection and electricity metering.

(zsd0921115@126.com)

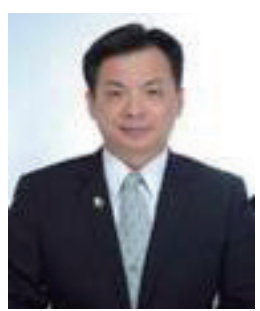

Chih-Cheng Chen has been a professor at Jimei University, China, since 2017. He became a member of IEEE in 2011 and a senior member in 2016. He earned his M.S. and Ph.D. degrees from the Department of Mechatronics Engineering, National Changhua University of Education. His research interests include AIoT technology and RFID applications.

(ccc@gm.cyut.edu.tw) 